\title{
Les futurs profils professionnels des médecins et des infirmiers dans la pratique ambulatoire et clinique
}

Groupe de travail «Profils

professionnels» * de l'ASSM

\footnotetext{
* Les personnalités suivantes ont fait partie du groupe de travail chargé d'élaborer ce rapport à l'intention du groupe de pilotage «La médecine en Suisse demain»: Dr Werne Bauer, Küsnacht (président); Prof. Anne-Françoise Allaz, Genève; Prof. Charles Bader, Genève; Barbara Gassmann, Berne; lic. oec. Pius Gyger, Zurich; Dr Jacques de Haller, Berne; Dr Romy Mahrer Imhof, Bâle; Dr Peter Tschudi, Bâle; Priska Wildhaber Isler, Küsnacht.
}

Correspondance:

ASSM

Petersplatz 13

CH-4051 Bâle

mail@samw.ch

\section{Mandat et manière de procéder du groupe de travail}

Le rapport «Buts et missions de la médecine au début du $21^{\mathrm{e}}$ siècle» met en évidence que les profils du médecin et du personnel soignant ont radicalement changé ces dernières décennies; ceux-ci peuvent, de ce fait, se sentir quelque peu déstabilisés dans leur identité professionnelle. Selon ce rapport, les professions médicales connaîtront à l'avenir des modifications profondes dans les définitions des différents rôles.

Le groupe de pilotage du projet «La médecine en Suisse demain» a accordé une grande importance à cette problématique et décidé d'en faire un des principaux points de la phase III du projet; à cet égard, il a chargé un groupe de travail du mandat suivant:

Dans un premier temps, le groupe de travail définit les rôles professionnels actuels (tant du point de vue interne qu'externe) au sein du système de santé (médecins et personnel soignant), esquisse les axes d'évolution probables, désigne les domaines conflictuels possibles et tente de définir un objectif prévisionnel pertinent.

Dans un deuxième temps, le groupe de travail définit les mesures à prendre dans le domaine de la formation et éventuellement dans d'autres domaines (p.ex. nouvelle répartition des rôles; relations publiques) afin $d^{\prime}$ atténuer les tensions et éventuellement d'approcher l'objectif.

Ce mandat est complexe et s'assigne un but élevé. Le groupe de travail est conscient des nombreux problèmes existant dans la délimitation et les points de jonction avec d'autres professions (p.ex. employé dans le secteur médical, assistante médicale). A cet égard, la relation entre les infirmières et infirmiers diplômés et les médecins peut être prise comme exemple, car elle est source de nombreux conflits; c'est pourquoi, le groupe de travail s'est limité dans son analyse et ses recommandations - conformément au mandat qu'elle a reçu - à ces deux groupes professionnels. Il a toujours orienté son travail vers ce qui est faisable et essayé de ne pas perdre de vue le lien avec la réalité. Le groupe de travail a placé au centre de ses réflexions les besoins du patient individuel tout en respectant les conditions nécessaires à une assistance médicale optimale de la population.

Le texte actuel est à considérer comme une base de discussion. Le rapport «Buts et missions de la médecine au début du $21^{\mathrm{e}}$ siècle», publié par l'ASSM, constitue le fondement de ce qui suit et est donc supposé connu. Il va de soi que les estimations et appréciations, relatives aux évolutions et à leurs conséquences, ne peuvent pas, au sein du groupe de travail, donner lieu à une complète unanimité et qu'il subsiste des divergences d'opinions. Toutefois, le texte résultant d'un travail commun, les membres du groupe de travail ont considéré qu'il n'était pas utile de mentionner de façon explicite toutes les divergences d'opinions.

Le groupe de travail définit le profil professionnel comme la description des activités et missions d'un groupe professionnel donné et la présentation du contexte ainsi que des conditions fondamentales requises à l'exercice de la profession en question: environnement social, conditions de travail, formations prégraduée et postgraduée. Un profil professionnel ne contient pas de catalogue détaillé des objectifs de formation.

Le groupe de travail définit l'identité professionnelle comme la spécificité éprouvée par les praticiens pour leur profession ainsi que les caractéristiques et distinctions de cette dernière. Le texte suivant n'aborde pas particulièrement la différenciation de ces notions.

Le groupe de travail considère son témoignage comme une base qui doit permettre un large débat et espère que celui-ci aura lieu dans le même esprit ouvert, constructif et tourné vers l'avenir, que celui qui a régné au sein du groupe.

\section{La médecine dans le contexte social}

Les médecins, infirmières et infirmiers ne travaillent pas en milieu protégé. Les évolutions sociales qui, du système de valeurs au système économique, touchent tous les domaines, ont une 
influence déterminante sur les conditions cadres de leur activité. Les tendances suivantes revêtent une importance toute particulière.

\section{La diversification croissante}

Le secteur de la santé connaît une mutation rapide et se diversifie toujours davantage: on trouve un nombre croissant de prestataires, des organisations et des modèles en concurrence, de nouvelles formes de prestations de soins, une variété de méthodes ainsi que de nouveaux systèmes de tarifications et de contrôles. De la même manière, la liberté de choisir son médecin est remise en question. La politique a une influence croissante sur tous les plans du système de santé, et, de la sorte, sur les profils professionnels. Les assureurs sont également devenus des acteurs importants bien décidés à défendre leurs intérêts.

On observe également une multiculturalité croissante, tant chez les patients que chez les collaborateurs.

L'élément-clé restera cependant inchangé: il existera constamment des personnes souffrantes qui auront besoin d'aide; la mission principale du secteur de la santé sera toujours de se préoccuper des personnes souffrantes et de leur procurer cette aide, de la meilleure manière possible.

\section{De I'assistance médicale au «marché médical»}

Les tensions augmentent entre le mandat de la santé publique, centré sur la personne et l'humain, et les facteurs économiques toujours plus dominants. Le secteur de la santé est de plus en plus influencé par des intérêts économiques, les pouvoirs du marché et les contraintes économiques.

S'il existe des sociétés/compagnies/firmes prospères qui parviennent à réaliser d'importants profits - des chaînes d'hôpitaux aux offres de technologie médicale, en passant par l'industrie pharmaceutique -, on est également confronté à de lourdes contraintes financières qui conduisent à une perplexité étendue et laissent poindre l'espoir qu'une solution aux problèmes pourrait se dissimuler dans la notion de «management».

Une attitude revendicative, visant le secteur de la santé et assortie des exigences correspondantes, est en train de gagner en intensité. L'attente croissante de voir se mettre en œuvre, de manière immédiate, parfaite et réussie, toutes les mesures se manifeste, entre autres, en proportion directe à la hausse des primes d'assurances. En même temps, on constate que les compétences de base de la population en matière de santé ainsi que sa capacité à gérer elle-même des petits troubles de santé diminuent à vue d'œil. En outre, la tendance à la médicalisation des problèmes économiques et sociaux se poursuivra.

\section{Féminisation}

Alors que la profession soignante passe depuis longtemps pour une "profession féminine classique», une évolution semblable se dessine depuis quelque temps en médecine. Actuellement, $60 \%$ des étudiants en médecine sont des femmes et cette proportion est toujours croissante. Cette «féminisation» de la profession aura des effets inévitables sur l'identité professionnelle.

\section{Flux d'informations}

Les informations disponibles se présentent de plus en plus souvent sous la forme d'une «avalanche» écrite ou électronique, ce qui rendra la tâche d'orientation difficile voire impossible au non-initié.

Pour atteindre les objectifs fixés aujourd'hui, au sens d'une «prise de décision partagée» ou de la responsabilisation du patient («patient empowerment»), l'évaluation de l'information par un professionnel de la sphère médicale est devenue nécessaire.

\section{Les transformations de I'activité professionnelle}

Nous constatons également avec une netteté croissante des transformations dans la pratique des professions médicales et infirmières. Outre le changement et le dynamisme qui y sont liés, on y trouve également des éléments qui freinent, entraînant actuellement, en Suisse, une image de coexistence qui évoque les mosaïques: prestations de soins d'un mode très traditionnel, et nombreux projets et modèles pilotes.

\section{De nouvelles professions émergent}

Les profils professionnels, bien connus et qu'on supposait clairement définis - des médecins généralistes, médecins spécialistes, infirmiers et autres personnes actives dans le secteur de la santé - tendent à se brouiller. De nouvelles professions surgissent et les limites avec celles qui existent déjà s'estompent partiellement. Dans le secteur de la santé, le premier interlocuteur et prestataire de divers examens et thérapies n'est plus nécessairement un médecin, comme cela était pratiquement toujours la règle jusqu'ici, exception faite de la médecine alternative.

\section{Travail en réseau}

Le travail d'équipe et la mise en réseau sont généralement considérés comme des modèles d'avenir. Cependant, notre pays connaîtra, long- 
temps encore, les cabinets individuels, les cabinets de groupe de petites dimensions ainsi que des formes d'organisations correspondantes dans le secteur des soins hors hôpital. Le souhait légitime de cultiver une relation personnelle et de longue date avec un médecin généraliste reste très solidement enraciné dans notre population, même si nombreux sont ceux - et, parmi eux surtout des personnes plus jeunes - qui tiennent vraiment à leur liberté de décider, au cas par cas, à qui s'adresser.

\section{Les formations pré- et postgraduée en mutation}

Les formations pré- et postgraduée sont également soumises à un changement; celui-ci est devenu, pour la première fois, particulièrement apparent sur le plan de la formation des professions médicales et infirmières, lors de l'introduction du modèle de Bologne. Les conséquences sont, pour l'instant, difficilement prévisibles, tandis que suivront des changements touchant également la structuration de la formation postgraduée.

\section{Les conditions de travail changent}

Il ne sera plus possible d'exiger des médecins et des infirmiers, dans leur activité normale, une astreinte à des horaires de travail dépassant ceux de professions (et de fonctions dirigeantes comparables) parmi celles qui nécessitent des services d'urgence, de nuit et de jours fériés.

On trouve d'ores et déjà des zones conflictuelles, de toutes sortes et à des degrés divers, qui se manifestent de manière de plus en plus prononcée sur les intersections suivantes:

- médecins spécialistes $><$ médecins de premier recours;

- médecins $><$ infirmiers $><$ autres professions de la santé;

- médecins, infirmiers indépendants $><$ hôpitaux, foyers, organisations Spitex;

a Le «disease management» permet aux patients, en concertation avec les médecins et d'autres soignants, de gérer de façon efficiente leur maladie chronique et d'en prévenir les complications par une adhésion au traitement, par l'évaluation régulière de signes vitaux, par une diète saine, par l'exercice et d'autres choix de style de vie.

b Le «case management» (gestion de cas) dans le domaine de la santé est un processus collaboratif impliquant l'assurance, le personnel soignant et le patient. Il vise en principe à assurer les besoins en santé du patient de la façon la plus efficiente et économique possible. connaissances et d'aptitudes, caractéristiques du profil professionnel; cela est réalisable grâce à un ensemble structuré qui englobe les formations pré- et postgraduée ainsi que les mesures d'accompagnement.

\section{Des lieux d'accueil accessibles}

Le premier interlocuteur du secteur de la santé n'est pas nécessairement un médecin.

Le lieu d'accueil préliminaire doit être une institution accessible, facile à contacter: cabinet de groupe, centre, établissement médico-social, urgences ou réseau, où il est possible d'effectuer toutes les clarifications et traitements non spécialisés; c'est aussi l'endroit depuis lequel s'effectue le transfert direct aux services compétents. Les centres d'appels, portails internet et pharmacies pourront également, bien que plus marginalement, assumer la fonction de lieu d'accueil préliminaire.

\section{Travail interdisciplinaire d'équipe}

Dans le futur, la répartition des tâches ne se conformera plus impérativement à des critères hiérarchiques, définis par le code déontologique ou liés à un titre officiel. Le travail interdisciplinaire d'équipe prend de plus en plus d'importance: l'activité des médecins se concentrera en premier lieu sur les domaines suivants: établissement de diagnostics et d'indications, traitement et conseils, «disease management ${ }^{\mathrm{a}}$ de cas complexes et méthodes spécialisées. Les infirmiers assumeront des tâches supplémentaires en matière de diagnostic, de thérapie et de «case management ${ }^{\mathrm{b}} \mathrm{qu}^{\prime}$ ils réaliseront de manière autonome. Certaines méthodes ou procédés techniques, comme par exemple la sonographie, l'endoscopie diagnostique ou les usages de laser, seront probablement de plus en plus appliqués par des non-médecins ou des médecins qui seront passés par une formation postgraduée spécifique restreinte. Pareille formation pourrait aussi, le cas échéant, déboucher sur l'attribution d'un certificat autre qu'un diplôme de médecin spécialiste.

Plus la spécialisation se développe et se ramifie, plus les généralistes, aux connaissances sans cesse actualisées en matière de méthodes et de technologies disponibles, prendront de l'importance. Leur mission est de placer des repères, le long des itinéraires servant au diagnostic et au traitement.

\section{L'orientation vers les besoins des patients}

Les profils professionnels devraient, en premier lieu, se conformer aux besoins des patients; d'autres puissants groupes d'intérêts économiques et politiques ne manqueront cependant pas de faire 
valoir leur influence. Celle-ci se répercutera avant tout à travers le financement, ce qui aura également des conséquences lors du financement des formations pré- et postgraduée. La formation postgraduée sera de plus en plus confrontée à l'exigence d'une structuration et d'un démarrage ciblé qui doit se produire le plus tôt possible. Cette exigence pourrait également être étendue à la phase de formation prégraduée; le modèle de Bologne propose dès maintenant les instruments nécessaires en soi.

\section{Un apprentissage de toute une vie}

La formation postgraduée doit être structurée et organisée de manière modulaire.

L'éventail des activités au début d'une carrière médicale sera de plus en plus rarement identique à celui des activités en fin de carrière. C'est pourquoi l'acquisition de nouvelles compétences doit être possible, au cours d'une carrière et au prix d'un investissement acceptable.

La remise de diplômes et les processus de sélection constituent des instruments pertinents pour contrôler les connaissances et les aptitudes; ils présentent, en même temps, le risque d'être manipulés, sous le couvert de l'assurance-qualité, par des groupes de pression qui visent à l'instauration d'un monopole ou d'une régulation de l'accès.

Une formation continue ciblée, toute la vie durant, et la disposition à assurer et promouvoir constamment la qualité sont des conditions natu- relles et préalables à l'exercice des professions du secteur de la santé. Le contrôle de la formation continue et de la qualité ainsi que l'élaboration d'instruments de contrôle de la qualité doivent reposer sur un consensus fondé sur la preuve («evidence-based»); ils ne peuvent pas dépendre exclusivement des prestataires de soins, d'une instance publique ou des assureurs.

\section{Le diagnostic et le traitement ne sont pas tout}

L'identité professionnelle des professions médicales suppose également un engagement actif dans le domaine de la santé publique (prévention et promotion de la santé), dans l'enseignement et la recherche ainsi que des connaissances de base en économie.

\section{Le futur profil professionnel des médecins, infirmières et infirmiers}

Le profil professionnel des médecins et infirmiers se laisse décrire sur trois plans et est composé des parties suivantes (fig. 1):

1. Les éléments communs de base, propres aux professions du secteur de la santé:

- une connaissance élémentaire de l'être humain et de ses fonctions;

- les principes éthiques et déontologiques et l'attitude de base qui en découle, au sens de «sciences humaines en médecine»;

- des aptitudes pratiques fondamentales,

Figure 1

L'identité professionnelle des médecins et des soignants peut se définir à trois différents niveaux: 1 . les éléments de base communs à toutes les professions de la santé (p.ex. les principes éthiques ou la capacité de communiquer avec les patients); 2. les éléments spécifiques à la profession ainsi que 3. les éléments spécialisés.

\begin{tabular}{|l|l|}
\hline Médecins & Infirmiers \\
\hline Spécialisations & Spécialisations \\
\hline $\begin{array}{l}\text { Eléments propres au profil } \\
\text { professionnel }\end{array}$ & Eléments propres au profil \\
\hline Eléments communs de base & \\
\hline
\end{tabular}


- une langue commune et la capacité adéquate à communiquer avec les patients et les autres professionnels;

- des connaissances fondamentales du système de santé suisse, des bases légales et du contexte économique;

- des connaissances de base en matière d'assurance qualité;

- un entendement en matière d'enseignement et de recherche.

L'enseignement de ces éléments de base est intégré dans les formations des groupes professionnels respectifs; des modules communs peuvent être envisagés.

2. Les éléments faisant partie des profils professionnels caractéristiques du groupe de tous les médecins et infirmiers: des connaissances spécifiques mais non spécialisées des fonctions normales et perturbées chez l'être humain; les aptitudes et techniques fondamentales nécessaires, telles que l'écoute active, l'observation, les techniques d'examen de base et de consultation médicale.

3. Les éléments particuliers des professions prises individuellement dans le domaine médical et des soins: compétences spécialisées, techniques ou autres, selon la fonction.

\section{Le futur profil professionnel des médecins}

\section{Missions et activités}

Au sein du système de santé suisse, le médecin occupe une fonction centrale: il est chargé - en association avec d'autres groupes professionnels de la prévention, du diagnostic et de la thérapie des troubles aigus de la santé, des thérapies palliatives et de la réhabilitation ainsi que des soins de longue durée en cas de maladie chronique. L'élément de base reste la relation personnelle et souvent de longue durée qui existe entre le médecin et son patient.

La profession de médecin comporte deux domaines d'activité majeurs: le premier concerne l'assistance médicale de base, assuré par le médecin généraliste, hospitalier ou rattaché à une institution médicale; le deuxième est le propre du spécialiste d'une méthode ou d'une technique déterminée, qu'il applique, dans le cadre d'un concept diagnostique ou thérapeutique, à un patient particulier. La division de la médecine en secteur ambulatoire et secteur hospitalier continuera, en outre, d'exister, bien que son caractère soit sans cesse appelé à se transformer: croissance du secteur ambulatoire, séjours hospitaliers de plus courte durée, interdépendance au niveau personnel.
Les deux groupes de médecins collaborent étroitement et pratiquent même, dans certains domaines, en concurrence avec d'autres professionnels de la santé.

Ces médecins - qui exercent au niveau de l'assistance médicale de base, ambulatoire (médecine générale) ou hospitalière (urgences, services et cliniques de médecine interne générale) - ont, généralement, suivi une formation postgraduée en médecine générale ou interne - pour obtenir, à l'avenir et compte tenu de la reconnaissance européenne, un titre de spécialiste en médecine interne générale - ou en pédiatrie.

De plus, il existe, au sein de nombreuses spécialités, une subdivision qui sépare l'assistance médicale de base et la médecine hautement spécialisée, comme, par exemple, chez les ophtalmologues qui pratiquent en cabinet et les chirurgiens du corps vitré ou de la rétine.

Les médecins généralistes sont des experts dans les domaines suivants: écoute active (anamnèse, «prise de décision partagée», consultation), diagnostic clinique et thérapie, appréciation et formulation des priorités dans les cas médicaux complexes, coordination de méthodes diagnostiques et de thérapies ainsi que dans l'établissement d'indications, dans le cas de transferts du patient à des spécialistes et des centres spécialisés.

En fonction de leur lieu de travail et de leur compétence personnelle, ils effectuent euxmêmes les mesures techniques indiquées (p.ex. échographies ou interventions légères de nature diagnostique et curative) ou ils orientent leurs patients vers un spécialiste au sein de leur équipe ou à l'extérieur de celle-ci, lorsqu'une mesure peut être mise en œuvre de manière plus efficace, économique ou compétente par un autre professionnel.

Ils prennent part au service d'urgence et consultent à domicile ou en institutions de soins. Les missions principales du généraliste sont la coordination, l'interprétation des résultats de l'analyse, l'établissement de bilans et la communication avec ses patients.

Le caractère attrayant de la profession provient du défi technique à relever dans la résolution de problèmes complexes et du rôle des relations, souvent de longue durée, aux patients et à l'équipe; à cela vient s'ajouter la position-clé - de par ses compétences décisionnelles - de cette activité au sein du système de la santé.

Les médecins spécialistes sont des professionnels de la plus haute compétence dans un domaine particulier. Il peut s'agir ici d'applications techniques - telles que, par exemple, les interventions chirurgicales spécialisées, les procédés invasifs 
diagnostiques ou la radio-oncologie - ou encore d'autres méthodes spécifiques, telles que les psychothérapies ou les traitements oncologiques.

Chez les patients dont le problème clinique est unique et prédominant, et pendant la durée du traitement par le médecin spécialiste, celui-ci peut également assumer la responsabilité médicale intégrale du patient. Les mesures prises par le spécialiste sont appliquées en étroite collaboration avec l'équipe généraliste de soins, chaque fois que l'on rencontre un ensemble de problèmes touchant la santé et parfois d'ordre social, psychosocial, liés aux soins ou d'une autre nature. L'activité du médecin spécialiste exige sa pleine compétence au sein de sa spécialité, qui impose toujours que les prestations soient fournies dans le cadre d'un concept global de traitement et de soins, curatifs ou palliatifs.

Le caractère attrayant de la profession est fondé sur le défi technique à relever - au moyen de méthodes modernes, exigeantes, technologiques en pleine évolution ou autres - et sur la contribution hautement efficace au diagnostic et à la thérapie, au sein d'une équipe ou d'un réseau; il faut ajouter à cela la conscience de pouvoir aider le patient de manière déterminante lorsque l'indication est correctement établie.

Toutes les professions médicales exigent que l'on accepte temporairement un engagement supérieur à la moyenne, en cas d'urgence ou lorsque des services de nuit et de jours fériés sont nécessaires.

\section{Environnement professionnel}

Les médecins ne peuvent véritablement s'épanouir - professionnellement et en conformité avec leur formation pré- et postgraduée - que moyennant un contexte et des conditions de travail adéquates.

La liberté de diagnostic et de l'orientation thérapeutique à donner - dans le cadre de normes professionnelles et techniques reconnues (directives, «exigences d'efficacité, d'adéquation et d'économicité») - reste une condition préliminaire de base à l'activité du médecin, que celui-ci exerce en pratique libre ou en qualité d'employé.

Des facteurs économiques, des conditions cadres et des arguments, pouvant aller jusqu'à la contrainte, caractérisent la médecine d'aujourd'hui. Sur leur lieu de travail, les médecins doivent pouvoir assumer de manière convaincante leur position et la défendre efficacement. Cela s'applique aux coûts des mesures prescrites mais aussi à la défense légitime des intérêts de chaque profession.
L'activité des médecins ambulatoires est également de plus en plus structurée à la manière d'un réseau qui peut être organisé sous forme d'équipe, au sein d'un centre ou décentralisé. La coopération avec les infirmiers a une importance capitale lorsqu'il s'agit des soins adéquats aux patients; il faut ajouter que cette collaboration n'est pas caractérisée par un rapport hiérarchique formel, mais bien par la question de savoir qui est compétent pour réaliser au mieux une tâche donnée. Certains suivis, consultations et mesures thérapeutiques peuvent être délégués de manière pertinente et autonome - dans le cadre d'un concept défini à des infirmiers, après établissement du diagnostic et choix de la thérapie par le médecin.

L'intégration de l'activité du médecin, au sein du travail d'équipe, doit conduire à un allégement des tâches administratives, de la gestion des ressources humaines, des commandes ainsi que d'autres travaux paramédicaux similaires; elle permet ainsi la concentration sur le contenu essentiel de la profession du médecin.

La rémunération du médecin doit correspondre à sa qualification professionnelle, aux responsabilités qu'il doit assumer et doit être conçue de manière à éviter les incitations indésirables. Il s'agit de prévenir les conflits d'intérêts, que ceuxci aillent vers un accroissement quantitatif ou vers un rationnement.

\section{Conditions préalables}

\section{à l'exercice de la profession}

La première condition à l'exercice de la profession de médecin est l'obtention du diplôme de fin d'études de médecine au niveau de la maîtrise universitaire ainsi que la réussite de l'examen fédéral. Celui-ci garantit un savoir de base commun à tous, la même langue et, autant que possible, une attitude de base uniforme.

Il est concevable - bien qu'encore incertain que, dans l'avenir, des diplômes spécifiques soient proposés au niveau de la maîtrise universitaire pour certains secteurs professionnels de la médecine. Il pourrait, par exemple, être question d'un diplôme particulier - pour la recherche ou des filières techniques qui auront été définies - à la manière de ce qui se fait depuis longtemps déjà pour la filière dentaire. Le même cursus et le même diplôme, valables pour tous, sont cependant nécessaires, y compris lorsque les soins vont au-delà d'un acte médical unique sur un patient précis.

La deuxième condition préalable réside dans une formation postgraduée diplômée, qui permette à une personne d'être capable d'exercer une activité autonome dans la spécialité en question. Il faut souligner l'importance de cette forma- 
tion - à la fois structurée et préparant de manière systématique au domaine d'activité choisi - qui doit être d'une exigence équivalente pour tous les titres de médecins spécialistes.

Il est également indispensable, au cours de la période de formation postgraduée, de développer la prise de conscience de la responsabilité personnelle, de favoriser la disposition à travailler en équipe et de promouvoir l'intérêt pour l'enseignement et la recherche. Par ailleurs, cette formation doit renforcer la conscience de la nécessité de se former continuellement («lifelong learning») et de procéder à une remise en question de la qualité des ses propres prestations (un ensemble d'attitudes que les anglophones qualifient de «professionalism»).

Des connaissances fondamentales en économie sont indispensables au travail de tous les jours et à une argumentation réussie, au même titre que l'aptitude à remettre son propre travail en question, à se former de manière ciblée et à participer à des projets concernant l'assurance-qualité.

Les mesures, visant la qualité et qui sont réclamées par une instance publique ou les assureurs, exigent une légitimation indiscutable.

Un système de formation postgraduée, flexible et structuré de manière modulaire, doit également permettre - au cours de l'existence et au prix d'un investissement en temps acceptable la réorientation professionnelle et l'acquisition des compétences nécessaires dans une spécialité voisine.

\section{Le futur profil professionnel des infirmiers}

Le profil professionnel de l'infirmier diplômé repose sur les éléments de base communs aux professions du secteur de la santé et sur la définition des soins professionnels, telle qu'elle est développée ci-dessous.

\section{Missions et activités}

Les personnes de tous âges, se trouvant dans les conditions de vie les plus diverses, peuvent être dépendantes des soins donnés par des infirmières et infirmiers qualifiés. L'enjeu de la prévention, pour sa part, est de promouvoir et de conserver la santé des individus et des familles et de prévenir les atteintes à la santé. Les malades ont besoin des interventions du personnel infirmier pour le traitement de leurs maladies. Ces personnes et leurs familles ont également besoin de soutien pour faire face aux répercussions des maladies et thérapies sur leur quotidien. L'activité englobe ici les mesures - au niveau de l'individu et de la famille - qui visent à recouvrer la santé, à surmonter la condition de la maladie et à accompa- gner le malade, lors de crises et au moment de mourir. Un groupe-cible précis ou bien encore la population toute entière peut constituer des champs d'activité supplémentaires pour les infirmiers. Il s'agit, dans ce cas, de développer des stratégies permettant de garantir des soins adaptés aux besoins et/ou des stratégies visant la promotion, à long terme, de la santé.

Les soins professionnels sont donnés au sein des cadres les plus divers du système de la santé: il faut penser ici au niveau communal (organisations Spitex, puériculture, cabinets de groupe, centres de santé), aux institutions très spécialisées (cliniques universitaires et spécialisées) ainsi qu'à la promotion de la santé/prévention (lieux de travail et écoles).

Les infirmiers qui bénéficient d'une formation généraliste au niveau du baccalauréat universitaire se chargent de l'assistance des patientes et patients en matière de soins hospitaliers, ambulatoires ou à domicile. Ils évaluent, en commun avec les patients et leurs proches, les besoins en soins et de prise en charge, planifient et mènent à bien les mesures, avant de procéder à l'évaluation de leur activité. Ce sont des experts en matière d'écoute active (anamnèse, "prise de décision partagée», consultation). Ils estiment les besoins de soutien qui se posent, d'une part, lors de l'application de thérapies et, d'autre part, dans les activités de la vie courante. Ils conseillent et accompagnent, de manière ciblée, les patients et les proches, afin d'accroître leur aptitude à se gérer, face à la maladie et à la thérapie, et de renforcer l'observance thérapeutique. En outre, ils conseillent les patients et les proches pour leur permettre de déceler et de réduire les risques pour la santé.

Les infirmiers jouent, lors de la constitution du plan de soins, un rôle important de coordination. Le but est ici de réduire, en même temps et autant que possible, les répercussions de la condition liée à la maladie sur le quotidien du patient. En plus de cela, les infirmiers planifient les soins, avec le concours de leurs collègues médecins et en impliquant d'autres professionnels (soins à domicile, service social, physiothérapie, etc.).

De par leur formation, les infirmiers sont en mesure de reconnaître les limites de leur compétence et de faire appel, au besoin, à d'autres professionnels de la santé, tels que le «médecin généraliste», les médecins spécialistes ou la conseillère en diététique (par exemple).

La manière de voir des infirmiers est davantage centrée sur la condition liée à la maladie que sur la maladie proprement dite, et s'oriente sur l'assistance qui permettra la maîtrise au quoti- 
dien des déficiences, résultant des perturbations de la santé. Leur raisonnement est influencé par leur lien avec le patient et son quotidien.

Les infirmiers qui se consacrent à une pratique clinique approfondie ont la possibilité de donner des soins qualifiés à un groupe de patients donnés (par exemple, les victimes d'insuffisance cardiaque, les patients en oncologie, les nouveaunés) ou dans le cadre d'une assistance précise (service ambulatoire/dispensaire, clinique spécialisée, Spitex, urgences, etc.). Les infirmiers qui ont une pratique approfondie bénéficient d'une formation au niveau de la maîtrise universitaire. Ils disposent de compétences élargies dans le domaine technique/médical et/ou en matière de conseil et d'accompagnement (par exemple, les soins gériatriques spécialisés ou les soins pédiatriques, le conseil aux diabétiques ou aux patients souffrant d'insuffisance cardiaque, la rééducation coronarienne). Ces infirmiers ont suivi une formation postgraduée dans des domaines spécialisés. Ils ont, en outre, été formés de manière à pouvoir introduire, au sein d'une institution, de nouveaux concepts de soins et former d'autres infirmiers, d'une manière fondée sur des preuves scientifiques. Ils sont en mesure de développer et d'instaurer de nouveaux concepts de soins et de conseil et utilisent, à cette fin, des méthodes scientifiques d'acquisition des connaissances.

\section{Environnement professionnel}

Les infirmiers peuvent exercer leur activité en qualité de salariés ou dans le cadre de leur cabinet personnel/d'une institution médicale. Ils agissent conformément aux normes éthiques et aux principes de qualité définis par la déontologie.

Face aux tiers, les infirmiers assument leur position de manière convaincante, lors de l'accompagnement des patients et de leurs proches, et les soutiennent efficacement. Ils sont, en outre, en mesure de défendre leurs propres intérêts professionnels - ainsi que ceux des patients.

Les infirmiers sont des partenaires essentiels à la coopération, au sein des réseaux du système d'assistance médicale. Ils sont qualifiés pour le travail en équipes. L'activité de celles-ci peut s'exercer en collaboration avec des médecins ou d'autres professions du secteur de la santé, au milieu de structures centralisées (hôpitaux, foyers, etc.) ou décentralisées (Spitex de la commune, établissement médico-social, réseau de soins). En même temps, cette coopération est orientée de manière à servir utilement les soins et l'accompagnement des patients et de leurs proches. En fonction des situations, les infirmiers vont assumer des tâches de coordination, d'organisa- tion ou d'exécution. Ils endossent des missions telles que la réalisation de soins, une fois que le médecin aura posé son diagnostic et prescrit la thérapie; en outre, ils assument les mesures de prévention, tout en se chargeant du conseil, de l'accompagnement à long terme des patients et de la réalisation du suivi.

Le travail en équipe doit être organisé de manière à permettre aux infirmiers de se concentrer sur le contenu essentiel de leur profession et d'être libérés des tâches administratives, de la gestion du matériel et des ressources humaines.

Les infirmiers fournissent souvent leurs prestations 24 heures sur 24 et 7 jours sur 7 . Les conditions cadres, relatives à l'exercice de la profession, sont à aménager afin que les infirmiers puissent proposer leurs services, d'une manière efficace, adéquate et autonome sur le plan économique et dans le respect des normes professionnelles en usage.

\section{Conditions préalables \\ à l'exercice de la profession}

Au niveau professionnel, un infirmier détient un diplôme de niveau baccalauréat universitaire. Cette formation le qualifie pour exercer sa profession, dans son orientation générale. Une spécialisation peut s'effectuer par la suite.

Des diplômes au niveau de la maîtrise universitaire sont proposés, qui permettent l'approfondissement des connaissances/compétences techniques dans des domaines professionnels particuliers; ils permettent également à l'infirmier de se consacrer à des groupes de patients spécifiques, aux besoins en conseils et en accompagnement particuliers. Les soignants titulaires d'une maîtrise universitaire peuvent assumer, de manière autonome, des fonctions à responsabilité dans ces spécialités.

Une formation postgraduée diplômée qualifie les soignants, en vue de l'exercice d'une activité autonome dans les spécialités, domaines d'activités ou techniques correspondants. Ces formations postgraduées doivent être orientées vers une activité professionnelle clairement décrite. Il faut, dans ce contexte, surveiller les normes et critères de qualité. Un système de formation postgraduée modulaire permet, au cours d'une carrière et au prix d'un investissement de temps acceptable, de se perfectionner, de se réorienter ou de se diriger vers un domaine professionnel voisin. Un tel système est évidemment perméable à de nombreuses autres disciplines. Des modules d'enseignement sont à développer - en coopération avec d'autres professions de la santé - et à fréquenter, lorsqu'il est question des 
bases professionnelles du secteur de la santé, ou que cela concerne la collaboration transdisciplinaire ou interprofessionnelle.

Les soignants avec un niveau baccalauréat universitaire exécutent les soins, en pratique, sur la base des preuves scientifiques. Pendant leur formation, ils apprennent à adopter une démarche et des méthodes de travail scientifiques. Leur collaboration à des projets de recherche est, de ce fait, souhaitable. En revanche, les étudiants au niveau de la maîtrise universitaire sont tenus de participer à des projets de recherche sous la direction d'un responsable. Une carrière scientifique autonome est possible après l'obtention d'un doctorat. Les soignants titulaires d'une formation de niveau maîtrise universitaire peuvent briguer ce titre.

\section{Conflits et conséquences}

La prise en charge optimale du patient doit être et rester le premier objectif dans le développement du système de la santé, en dépit de toutes les mutations de la société, de l'activité professionnelle et des conditions cadres.

Les modifications des identités professionnelles, telles que nous les esquissons et les attendons, se réaliseront en partie d'elles-mêmes, sous la pression des forces de l'innovation et du marché.

Toutefois, des mesures directrices et de soutien seront nécessaires afin de les orienter dans le sens considéré par le groupe de travail comme pertinent et conforme aux besoins des patients. Ces mesures peuvent avant tout débuter dans les domaines de la formation pré- et postgraduée, l'aménagement des modèles de travail, les certifications et les rétributions.

Les développements supposés présentent un potentiel conflictuel considérable, mais il est plus probable qu'on assiste à une aggravation des litiges préexistants qu'à l'apparition de tensions ou de conflits d'intérêts totalement neufs.

Il est avant tout question, ici, des relations entre les spécialistes et les généralistes, entre les médecins et les soignants et les autres professionnels du système de la santé, entre les secteurs hospitalier et ambulatoire, entre les professionnels indépendants et les institutions et entre les prestataires de soins et les prestataires financiers.

Les profils professionnels - tournés vers l'avenir - devraient, dès lors qu'ils sont acceptés et mis en application, améliorer la collaboration, désamorcer la problématique des points de liaisons et accroître l'efficience des prestations. Pour le moment cependant, il se pourrait que certains groupes défendent farouchement les rôles et modèles traditionnels, parce qu'ils craignent de faire partie des perdants face au changement.

Sans entrer dans les détails, le groupe de travail distingue, par conséquent, les effets et mesures suivants, susceptibles d'aider à désamorcer les conflits potentiels et à ne pas laisser la mutation des profils professionnels tout simplement «se produire», mais bien à la structurer activement, si cela est, d'une quelconque manière, possible.

\section{Conséquences pour les deux groupes professionnels: médecins et infirmiers}

- Il est nécessaire de redéfinir les compétences et responsabilités des médecins et infirmiers, au sein des groupes professionnels.

Des directives acceptées de façon générale peuvent vraisemblablement être élaborées pour une partie de cette définition; l'autre partie, cependant, doit, pour être pertinente, être élaborée et établie à l'intérieur de chaque institution prise séparément (hôpital, service ambulatoire/dispensaire, centre, cabinet de groupe, réseau). Certains domaines nécessitent néanmoins des adaptations légales préalables (par exemple pour une compétence élargie du règlement concernant les infirmiers).

- La formation au niveau baccalauréat universitaire procurera, à l'avenir, dans le sens d'un tronc commun, les mêmes éléments communs de base (principes, savoir fondamental, aptitudes, vocabulaire: cf. plus haut) aussi bien aux médecins qu'aux soignants. Ces modules communs doivent être élaborés.

- De nouveaux modèles d'activité transdisciplinaire en équipe (HMO, nouveaux modèles de «managed care», cabinets de groupes transdisciplinaires, collaboration décentralisée en réseau, etc.) - au sein desquels les professions individuelles peuvent être installées en fonction de leurs compétences de base - doivent être encouragés (cf. plus haut). Des mesures de régularisation prises au niveau fédéral ainsi que de nouvelles offres d'assurance doivent être soutenues et non pas freinées.

Pour les modèles visant à améliorer la collaboration interdisciplinaire, il convient d'élaborer des conditions cadres qui soient aussi peu restrictives que possibles et qui n'entraînent pas un surplus de travail administratif.

- Les associations professionnelles sont chargées d'introduire les mesures nécessaires et d'assurer un dialogue constructif.

L'esprit conservateur doit faire place à la conviction que le système de la santé a besoin de nouveaux modèles et concepts qui doivent 
être structurés activement, testés et constamment améliorés.

\section{Conséquences pour la profession de médecin}

- Les contenus d'enseignement aux niveaux baccalauréat universitaire et maîtrise universitaire sont à adapter régulièrement aux exigences de la société et de la profession.

La formation des médecins, dont le but professionnel est l'activité clinique, reste, en essence, la même pour tous, aux niveaux baccalauréat universitaire et maîtrise universitaire. Des filières particulières de formation sont concevables au niveau de la maîtrise universitaire pour les étudiants dont le but professionnel est la médecine théorique ou pour des professions très spécifiques, d'orientation technique.

- Dans les cas complexes de troubles de la santé, il faudra développer des modèles de «disease management» et définir des itinéraires de patients - servant au diagnostic et à la thérapie - pour lesquels un médecin généraliste sera, en principe, responsable.

C'est lui qui devra assumer en priorité la mission de coordination; il expose au patient les différentes mesures à envisager et en discute avec lui. Ces mesures seront exécutées par des spécialistes compétents faisant partie de l'équipe ou non (p.ex. infirmier, assistant, spécialiste, institution spécialisée).

- La formation postgraduée est structurée dès le commencement et a la même valeur pour toutes les spécialités.

Des cursus clairement définis doivent permettre aux postulants de se décider en faveur d'une spécialité; un tel choix doit être fait dès que possible au commencement de la formation postgraduée.

- Il est nécessaire de disposer d'un nombre suffisant de places accréditées de formation postgraduée dans les hôpitaux ou les cabinets et d'assez d'emplois à temps partiel (en particulier pour les femmes qui suivent une formation postgraduée).

\section{Conséquences pour la profession d'infirmier}

- Le baccalauréat universitaire doit devenir également en Suisse alémanique et au Tessin - le diplôme normal du personnel soignant (en vigueur en Suisse romande depuis 2002). Il convient de promouvoir des possibilités de formation pouvant mener à la maîtrise universitaire ou au doctorat.

- Les contenus d'enseignement sont à adapter, en accord avec les médecins et compte tenu du futur tronc commun dans la formation.
A l'avenir, la formation au niveau du baccalauréat universitaire transmettra, aussi bien aux médecins qu'au personnel infirmier - en plus des connaissances spécifiques à leur domaine -, des éléments de base communs (mêmes modules pour les principes, les connaissances de base, les aptitudes, la langue: voir plus haut).

- Il faut intensifier le recrutement de la relève, en insistant sur les larges débouchés professionnels offerts.

\section{Conséquences pour la politique, les assureurs} et les organisations professionnelles

- En conséquence, les dispositions légales doivent être révisées de manière à éviter que les réglementations des délégations médicales et les réglementations tarifaires liées à des réalités institutionnelles ne constituent un obstacle à une répartition optimale des compétences et des responsabilités. Les lois et règlements doivent être ajustés de façon à rendre possible l'adaptation des compétences et des responsabilités, telle qu'elle est demandée dans ce rapport (p.ex. art. 25, al. 2a, Art. 35 LAMal, Art. 7 OPAS, Art. 49 OAMal). [Ainsi, les soignants auront aussi la possibilité d'exercer leur activité en tant que prestataire indépendant à la charge de l'assurance dépendance.]

Ainsi, l'activité des soignants en tant que prestataires sera également soumise à l'assurance-maladie obligatoire. A cet égard, on trouve dans l'art. 35 LAMal la liste des prestataires dont les prestations sont payées par l'assurance-maladie obligatoire. Les infirmiers indépendants doivent également figurer sur cette liste.

- En conséquence, les modèles tarifaires existants doivent être adaptés et rendus plus flexibles, de manière à trouver la base financière nécessaire à la nouvelle répartition des compétences et des responsabilités demandée dans ce rapport.

Cela signifie, par exemple, que certaines prestations seront facturées forfaitairement ou par groupe de prestations par une organisation de la santé.

- Il faut promouvoir activement les modèles de réseaux et les soins coordonnés («coordinated care»), de manière à ce que la nouvelle répartition des compétences et des responsabilités, telle qu'elle est demandée dans ce rapport, trouve également sa correspondance au niveau structurel.

Cela signifie que toutes les parties prenantes assument leur responsabilité envers le système 
de santé et élaborent de nouveaux modèles d'assurance, qui soient, d'une part, conformes au marché, mais qui, d'autre part, ne freinent pas l'optimisation de la prestation centrée sur le patient par des équipes transdisciplinaires.

- Les organisations professionnelles doivent absolument mener des campagnes d'information et de persuasion de la classe politique, des assureurs, des leaders d'opinion, des autres décisionnaires et de l'opinion publique.

Dès lors, il est indispensable que les membres des organisations professionnelles soutiennent les réflexions de principes concernant leur identité future et les concepts détaillés qui doivent être élaborés.

\section{Recommandations}

\section{au comité de direction de I'ASSM}

- Elaboration d'une «Charter for Coordinated Care»;

- Organisation d'une manifestation sur le thème de l'«Identité professionnelle des médecins et du personnel infirmier» (public ciblé: médecins, infirmiers, politiciens, assureurs, journalistes).

Littérature sélectionnée (par ordre chronologique)

- Kesselring A, de Geest S. Activité soignante et médecine: sœur et frère siamois? bulletin ASSM. 2002;(3):1-3.

- Bürgi H, Bader C, Bloch R, Bosman F, Horn B, Im Hof V, et al. Swiss Catalogue of Learning Objectives for Undergraduate Medical Training. Berne: SMIFK; 2002.

- CRS. Formation des infirmières et infirmiers diplomés. Prescriptions relatives. Berne: CRS; 2002.

- Kaufmann C. Medizin im Aufbruch: Neues Berufsbild, neues Gesetz, neue Ausbildung. www.unipublic. unizh.ch/magazin/gesundheit/2002/0595.

- Nordt C. Strukturwandel der medizinischen Grundversorgung: Ursachen und Wirkung der ärztlichen Arbeitszufriedenheit in unterschiedlichen Praxismodellen. Auszug (Zusammenfassung) aus Dissertation. Philosophische Fakultät, Universität Zürich; 2003.

- Charte du professionnalisme médical. Bull Méd Suisses. 2003;84(45):2350-2

- Spichiger E, et al. Professionelle Pflege - neu definiert: Zwei Kernsätze und acht Ergänzungen. Krankenpflege. 2004;98(8):20-3.
- Bovier P, Bouvier Gallacchi M, Goehring C, Künzi B. Santé des médecins de premier recours en Suisse. PrimaryCare. 2004;4(47):941-7.

- Buchan J, Calman L. Skill-Mix and Policy Change in the Health Workforce: Nurses in Advanced Roles. OECD Health Working Papers 17. Paris: OECD; 2005.

- Gassmann B. Collaboration ou concurrence? Bull Méd Suisses. 2005;86(29/30):1764.

- Grüninger U. Braucht es den Hausarzt noch? PrimaryCare. 2005;5(3):58.

- Simoens S, Villeneuve M, Hurst J. Tackling Nurse Shortages in OECD Countries. OECD Health Working Papers 19. Paris: OECD; 2005.

- de Haller J. Gibt es bald keine Hausärzte mehr? (interview). NZZ am Sonntag, 27.11.2005.

- Woodroffe E. Nurse-led general practice: the changing face of general practice? Br J Gen Pract. 2006; 56:632-3.

- Beer JH, Kaspar K. Ärztlicher Grundversorger als Berufsziel. NZZ, 14.3.2006.

- Barr M, Ginsburg J for the Health and Public Policy Committee of the American College of Physicians. The Advanced Medical Home. A Policy Monograph. Washington: ACP; 2006.

- Stiftung Careum (Hrsg.). Grenzen aufheben Thesen zur Zukunft der Ausbildung für Gesundheitsberufe. Zürich: Stiftung Careum; 2006.

- Freudenheim M. Attention Shoppers: Low Prices on Shots in Clinic. New York Times, 14.3.2006.

- Siegrist M, Orlow P, Giger M. Formation postgraduée et situation professionnelle vues par les médecins-assistants. Bull Méd Suisses. 2006;87(10): 379-86.

- Simoens S, Hurst J. The Supply of Physician Services in OECD Countries. OECD Health Working Papers 21. Paris: OECD; 2006.

- FMH. Oui aux réseaux et à la qualité - oui au «Managed Care». Thèses de la FMH concernant deux thèmes d'actualité essentiels du domaine de la santé - l'essentiel en bref. Bull Méd Suisses. 2006;87(36):1535-9.

- Weinberger SE, Smith LG, Collier VU; Education Committee of the American College of Physicians. Redesigning training for internal medicine. Ann Intern Med. 2006;144(12):927-32.

- Sachverständigenrat zur Begutachtung der Entwicklung im Gesundheitswesen, D. Kooperation und Verantwortung. Voraussetzungen einer zielorientierten Gesundheitsversorgung. Kurzfassung, 106 Seiten. Bonn; 2007.

- SSMI. A Proposal for Coordinated Care. Policy Monograph. Bâle: JGIM; 2007. 